\title{
The natural history of milk allergy in an observational cohort
}

\author{
Robert A. Wood, MD ${ }^{a,}{ }^{*}$, Scott H. Sicherer, MD ${ }^{b},{ }^{*}$, Brian P. Vickery, MD $^{c}$, Stacie M. Jones, \\ MD $^{d}$, Andrew H. Liu, MD $^{e}$, David M. Fleischer, MD $^{e}$, Alice K. Henning, $\mathbf{M S}^{f}$, Lloyd Mayer, \\ $\mathbf{M D}^{\mathrm{b}}, \mathbf{A}$. Wesley Burks, $\mathbf{M D}^{\mathrm{c}}$, Alexander Grishin, $\mathbf{P h D}^{\mathrm{b}}$, Donald Stablein, $\mathbf{P h D}^{\mathrm{d}}$, and Hugh A. \\ Sampson, MD \\ aDepartment of Pediatrics, Johns Hopkins University School of Medicine, Baltimore \\ bDepartment of Pediatrics, Mount Sinai School of Medicine, New York \\ 'Department of Pediatrics, University of North Carolina, Chapel Hill \\ 'Department of Pediatrics, University of Arkansas for Medical Sciences and Arkansas Children's \\ Hospital, Little Rock \\ eDepartment of Pediatrics, National Jewish Health, Denver \\ fEMMES Corporation, Rockville
}

\section{Abstract}

Objective-There are few studies on the natural history of milk allergy. Most are single-site and not longitudinal, and these have not identified a means for early prediction of outcomes.

\begin{abstract}
Methods-Children aged 3 to 15 months were enrolled in an observational study with either (1) a convincing history of egg allergy, milk allergy, or both with a positive skin prick test (SPT) response to the trigger food and/or (2) moderate-to-severe atopic dermatitis (AD) and a positive SPT response to milk or egg. Children enrolled with a clinical history of milk allergy were followed longitudinally, and resolution was established by means of successful ingestion.
\end{abstract}

\begin{abstract}
Results-The cohort consists of 293 children, of whom 244 were given a diagnosis of milk allergy at baseline. Milk allergy has resolved in 154 (52.6\%) subjects at a median age of 63 months and a median age at last follow-up of 66 months. Baseline characteristics that were most predictive of resolution included milk-specific IgE level, milk SPT wheal size, and AD severity (all $P<.001$ ). Baseline milk-specific $\mathrm{IgG}_{4}$ level and milk $\mathrm{IgE} / \mathrm{IgG}_{4}$ ratio were not predictive of resolution and neither was expression of cytokine-inducible $\mathrm{SH} 2$-containing protein, forkhead box protein 3, GATA3, IL-10, IL-4, IFN- $\gamma$, or T-bet by using real-time PCR in CD25-selected, caseinstimulated mononuclear cells. A calculator to estimate resolution probabilities using baseline milk IgE level, SPT response, and AD severity was devised for use in the clinical setting. Conclusions: In this cohort of infants with milk allergy, approximately one half had resolved over 66 months of follow-up. Baseline milk-specific IgE level, SPT wheal size, and AD severity were all important predictors of the likelihood of resolution.
\end{abstract}

\section{Keywords}

Milk allergy; natural history; food allergy; $\operatorname{IgE}$

\footnotetext{
(C) 2012 American Academy of Allergy, Asthma \& Immunology

Corresponding author: Robert A. Wood, MD, CMSC 1102, Johns Hopkins Hospital, 600 North Wolfe St, Baltimore, MD 21287. rwood@jhmi.edu.

These authors contributed equally to this manuscript.

Disclosure of potential conflict of interest:

The rest of the authors declare that they have no relevant conflicts of interest.
} 
Milk allergy is the most common food allergy in young children, with prevalence rates estimated in the range of $2 \%$ and $3 \% .{ }^{1,2}$ Although the natural history of milk allergy is generally favorable, with the majority of children showing resolution during childhood, prior studies have yielded widely varying results as to the rate of resolution. ${ }^{3-16}$ A recent study suggested that the natural history of milk allergy might have changed over time, with slower rates of resolution and a higher proportion of children with disease persisting into adolescence and even adulthood. ${ }^{15}$ Although these changes might be real, most differences between studies are more likely related to both study design and the specific population under investigation. For example, studies of the general population, ${ }^{11}$ especially if oral food challenges are performed at regular intervals, are more likely to demonstrate earlier resolution than studies of tertiary referral populations. $3,9,10,15$

The Consortium of Food Allergy Research enrolled infants with likely egg or milk allergy but without previously known peanut allergy in an observational study to address the immunologic, genetic, and environmental factors that affect the natural course of food allergy. ${ }^{17}$ The primary aim of this analysis was to assess the natural history of milk allergy in the infants enrolled in this cohort with a diagnosis of milk allergy, with a particular focus on the clinical factors predicting the resolution of milk allergy over the first 5 years of life.

\section{METHODS}

\section{Subjects, study definitions, and procedures}

The subjects of this study are a subset of a larger cohort of 512 infants originally enrolled at 3 to 15 months of age at 5 sites: Mount Sinai School of Medicine, New York, New York; Duke University Medical Center, Durham, NC; Johns Hopkins University School of Medicine, Baltimore, Maryland; National Jewish Health, Denver, Colorado; and Arkansas Children's Hospital, Little Rock, Arkansas, as described previously ${ }^{17}$; the North Carolina subjects moved with the investigative team from Duke to the University of North CarolinaChapel Hill in March 2012. Enrollment criteria for the whole cohort were designed to obtain atopic children with likely egg or milk allergy at risk for peanut allergy but without current peanut allergy. Briefly, enrollment required either (1) a history of a convincing immediate allergic reaction to cow's milk (and/or egg) and a positive skin prick test (SPT) response (3 $\mathrm{mm}$ larger than that elicited by the negative control) to cow's milk (and/or egg, if the clinical reaction was to egg) and/or (2) moderate-to-severe atopic dermatitis (AD) and a positive SPT response to milk, egg, or both.

The subgroup of children in the current study had a diagnosis of milk allergy at the time of enrollment or acquired this diagnosis after enrollment with no prior evidence of tolerance of milk (eg, enrollment diagnosis was uncertain). Study procedures were reviewed and approved by a National Institute of Allergy and Infectious Diseases Data Safety Monitoring Board and by local institutional review boards, and written signed consent forms were obtained.

Participants were considered to have milk allergy if they had either (1) a positive physiciansupervised oral food challenge result or a convincing reaction (defined by symptoms within an hour of isolated ingestion that included at least urticaria and/or angioedema, difficulty breathing, wheezing, throat tightness, and/or vomiting) and sensitization to milk (milkspecific IgE level $\searrow 0.35 \mathrm{kU} \mathrm{A}_{\mathrm{A}} / \mathrm{L}$ and/or SPT response $>3 \mathrm{~mm}$ ) or (2) a flare of AD associated with milk ingestion along with a milk-specific IgE level of greater than $5 \mathrm{kU} \mathrm{A}_{\mathrm{A}} / \mathrm{L},{ }^{18}$ which is greater than $95 \%$ predictive of milk allergy in infants. Reactions to goat's or sheep's milk were also considered evidence of cow's milk allergy. Subjects were considered milk tolerant if they ingested whole uncooked milk products (milk, yogurt, or ice cream) in serving size 
quantities without symptoms either during physician-supervised oral food challenges or after introduction at home. Dietary ingestion of products with extensively heated milk (baked milk, for example as an ingredient in a muffin) was queried but was not considered evidence of resolved milk allergy.

Dietary, medical, and social histories were obtained by using questionnaires completed during enrollment interviews. A diagnosis of asthma and allergic rhinitis was based on parental report or parental report of a physician's diagnosis. A diagnosis of other food allergies included per-protocol definitions for egg and peanut, ${ }^{17}$ whereas for other foods, this was based on a clinical diagnosis by a study physician.

Diagnosis of $\mathrm{AD}$ required pruritus and an eczematous rash (acute, subacute, or chronic) with typical morphology and age-specific patterns, a chronic or relapsing history, atopy (personal history, family history, or both or IgE reactivity), and xerosis. AD severity was graded based on criteria previously described and published by Rajka and Langeland. ${ }^{19}$ Briefly, the AD severity was graded as mild, moderate, or severe by using the following parameters (see Table E1 in this article's Online Repository at www.jacionline.org) ${ }^{19}$ to compute a score summation: (1) extent of disease (by "rule of nine" based on the proportion of body surface area with active disease), (2) course of disease (defined by history as $>3$ months in remission in the past year, $\mathfrak{S}$ months in remission but not continuous, or continuous remission over the past year), and (3) intensity of disease (defined as mild itch rarely disturbing sleep, severe itch usually disturbing sleep, or intermediate itch/sleep disturbance), each on a 3point scale. Summation scores of 3 to 4 indicated mild disease, 5 to 7 indicated moderate disease, and 8 to 9 indicated severe disease. Atopic disease history in parents of the enrolled infants was based on previously published definitions and was recorded by parental report. ${ }^{20}$

The study design includes evaluations, care for food allergy, and instructions on dietary management that were uniform among the 5 clinical centers and reflect practice parameters for $\mathrm{AD},{ }^{21}$ food allergy, ${ }^{22}$ and the American Academy of Pediatrics recommendations for allergy prevention published in 2000 to maintain uniformity and an observational approach. ${ }^{23}$ Participants were evaluated in person at enrollment, 6 months, 12 months, and yearly thereafter, with additional telephone follow-up between each visit and instructions to contact the study site for any allergic reactions, at which time additional details were obtained. ${ }^{24}$

\section{SPTs}

SPTs were performed with the GreerPick (Greer Laboratories, Lenoir, NC), with participants avoiding antihistamines for at least 5 half-lives of the specific agent. Tests were performed on the infant's back, and at 15 minutes, the wheal was outlined in pen and transferred by tape to paper. The size of the longest diameter and its longest perpendicular were averaged. An SPT score was computed by subtracting the saline control measure, and a positive SPT response was defined by a score of $3 \mathrm{~mm}$ or greater. Tests were considered reliable if the wheal of the negative control (50\% glycerin-saline) was $3 \mathrm{~mm}$ or smaller and wheal size elicited by the histamine control was at least $3 \mathrm{~mm}$ larger than the wheal size elicited by the negative control. All sites used the same lot of reagents, and training was performed to ensure consistency. The cow's milk extract was obtained from Greer (catalog no. F293).

\section{Serum milk-specific $\lg E$ and $\operatorname{lgG}_{4}$ levels}

The concentration of specific IgE antibody to milk was measured from plasma at a central laboratory (Mount Sinai) by using the Phadia (now Thermo Fisher Scientific, Waltham, Mass) ImmunoCAP system and reported in kilounits of allergen per liter. A level of 0.35 
$\mathrm{kU}_{\mathrm{A}} / \mathrm{L}$ or greater was considered positive. The concentration of $\mathrm{IgG}_{4}$ antibodies to milk was also measured from plasma samples by using the Phadia ImmunoCAP system. The detection limit for $\mathrm{IgG}_{4}$ is $0.07 \mathrm{mg} / \mathrm{L}$.

\section{Mononuclear cell stimulation and PCR analysis}

PBMC isolation was performed with Ficoll-Paque density gradient centrifugation, and cultures were performed at each clinical site on fresh venous blood samples, as previously described. ${ }^{1}$ Briefly, 4 million cells per condition were cultured for 48 hours in AIM-V serum-free media (Invitrogen, Carlsbad, Calif) with purified $\alpha-, \beta$-, and $\kappa$-caseins (50 $\mu \mathrm{g}$ each/L), and control stimulations were performed with medium alone (negative) and antiCD3/anti-CD28 beads (positive). At the end of the culture period, cells expressing CD25 were enriched by means of selection with anti-CD25-coated paramagnetic beads, according to the manufacturer's protocol (Miltenyi Biotech, Bergisch Gladbach, Germany). Pilot experiments demonstrated approximately 10 -fold enrichment of $\mathrm{CD} 25^{+}$cells, with $70 \%$ to $80 \%$ of selected cells coexpressing CD3, CD4, and CD25, as measured by using flow cytometry. The entire selected fraction of cells was immediately lysed in RLT buffer (Qiagen, Hilden, Germany) and stored at $-80^{\circ} \mathrm{C}$ until RNA purification. The quantitative PCR was carried out in the central laboratory according to the in-house established protocol by using SYBR Green I fluorescence detection in a 384-well plate on the ABI 7900 (Applied Biosystems, Foster City, Calif). Raw PCR analysis and annotation were performed on coded samples. The cycle threshold $(\mathrm{Ct})$ number was set by software, with confirmation and adjustment as necessary to define the threshold of linear amplification. For the gene expression data, $\Delta \Delta \mathrm{Ct}$ was calculated by subtracting the RPS9 reporter gene $\mathrm{Ct}$ value and then normalizing by subtracting the standardized medium control response. Negative values indicate relatively higher activity with a unit score change corresponding to a doubling. Nondetected genes were arbitrarily assigned a Ct value of 40 .

\section{Statistical analysis}

Time to resolution of milk allergy was measured with age as the time metric. Although the time of allergy diagnosis varied depending on when food introduction and diagnostic testing were performed, each subject's first definitive diagnosis was positive for milk allergy. Those subjects $(\mathrm{n}=12)$ who had resolution of their milk allergy before 15 months were assigned a value of 15 months. Because enrollment continued through 15 months, this permitted the use of fixed covariate baseline modeling in all participants.

Distributional differences in baseline variables were compared with the $\chi^{2}$ statistic. Proportional hazards regression models were fit to examine covariates for their effect on the hazard or risk function. ${ }^{25}$ The estimated survival distribution was calculated from the relative hazard $(\mathrm{RH})$, which is the exponentiated sum of the linear combination of the products of the parameter estimates with their respective clinical characteristics. The common underlying empiric cumulative hazard function $\lambda(t)$ is estimated with a step function, and the resolution curve is estimated as follows:

$$
1-\exp (-\mathrm{RH} * \lambda[\mathrm{t}])
$$

In this article hazard refers to the risk of a beneficial event, namely allergy resolution, and variables are structured so that large relative hazard values are associated with increased chance of allergy resolution. Model prediction capability for baseline variable models was summarized with the $\mathrm{C}$ index, a concordance measure of predicted and observed responses related to the Kendall rank correlation $\tau$ value. ${ }^{26,27}$ This measure extends the binary end point assessment of the area under the receiver operating characteristics curve to use with 
time-to-event data. The $\mathrm{C}$ index ranges up to 1 , and a value of 0.5 indicates no predictive ability. Time-varying clinical covariate analyses used the most recent available assessment in the model, and nonproportional hazards were examined by fitting linear and spline function interactions with time. For real-time PCR variables, an additional assessment of change from baseline was performed with scores of -1 when 4 or fewer doublings occurred relative to baseline, 1 when 4 or more doublings relative to baseline occurred, and 0 otherwise. Reported $P$ values are 2-tailed, when applicable, and SAS 9.2 (SAS, Institute, Cary, NC) and R software were used for computations.

\section{RESULTS}

Of the 512 enrolled infants, the cohort with milk allergy consisted of 293 children, of whom 244 were given a diagnosis of milk allergy at baseline. Among the remaining 49 children, the diagnosis was categorized as uncertain at their entry visit, but milk allergy was subsequently confirmed. Key baseline characteristics are summarized in Table I. A majority (178/293) of subjects were enrolled between 6 and 12 months of age; 192 were male, and 101 were female. AD was present in 261 subjects and was categorized as mild in 34 , moderate in 146, and severe in 81 . Twenty-four infants were given a diagnosis of milk allergy based on $\mathrm{AD}$ criteria, whereas the remainder had a history of an acute reaction to milk. One hundred seventeen (39.9\%) subjects were also given diagnoses of other food allergies at their initial evaluation, and another 74 (25.3\%) had other food allergies over the period of observation.

One hundred fifty-four (52.6\%) of the 293 participants have now resolved their milk allergy, with a median age of resolution of 63 months and a median age at last follow-up of 66 months (Fig 1). All but 6 subjects had follow-up beyond 4 years of age. Resolution was defined by means of oral food challenge in 56 subjects and by successful home introduction of uncooked milk products in 98 subjects. At the 5-year time point, 32 (20.6\%) of 155 subjects with unresolved allergy reported tolerating at least some baked milk products, whereas 7 reported reactions to ingestion of baked milk products.

Additional baseline characteristics of the cohort, comparing those with and without milk allergy resolution, are presented in Table I. The baseline characteristics that were most predictive of milk allergy resolution included milk-specific IgE level, milk SPT wheal size, and AD severity. Specifically, highly significant differences $(P<.001)$ in the rate of resolution were noted when comparing those subjects with baseline milk-specific IgE levels of less than $2 \mathrm{kU}_{\mathrm{A}} / \mathrm{L}, 2$ to $10 \mathrm{kU}_{\mathrm{A}} / \mathrm{L}$, and $10 \mathrm{kU}_{\mathrm{A}} / \mathrm{L}$ or greater (Fig 2). For example, greater than $70 \%$ of those in the lowest milk-specific IgE category had resolved milk allergy compared with only $23 \%$ of those in the highest category. Significant differences $(P<.001)$ in resolution were also predicted by baseline SPT results, as shown in Fig 3, which represents a comparison of subjects with wheal sizes of less than $5 \mathrm{~mm}, 5$ to $10 \mathrm{~mm}$, and greater than $10 \mathrm{~mm}$. In addition, marked differences in resolution $(P<.001)$ were detected when comparing those infants presenting with milk allergy who had no or mild AD with those with moderate-to-severe AD (Fig 4). However, baseline milk-specific $\mathrm{IgG}_{4}$ levels were not at all predictive of resolution (see Fig E1 in this article's Online Repository at www.jacionline.org), and the milk-specific $\mathrm{IgE} / \mathrm{IgG}_{4}$ ratio did not add further insight to the analyses (data not shown).

Cox regression analyses were conducted to further define the effects of these baseline variables on milk allergy resolution (Table II). Again, milk-specific IgE level, SPT wheal size, and AD severity stand out as the most important factors predicting resolution of milk allergy. For example, when analyzing milk-specific IgE level as a categorical variable, subjects with baseline levels of less than $2 \mathrm{kU}_{\mathrm{A}} / \mathrm{L}$ have a 5.74-fold increased hazard ratio of 
resolving their allergy versus those with levels of greater than $10 \mathrm{kU} / \mathrm{A}$. This analysis also revealed a nonsignificant trend toward higher rates of resolution in female versus male subjects. Other variables, such as parental atopic history, education, income, and presence of siblings, were examined, and none were statistically significant predictors (data not shown).

Serial measurements of milk-specific IgE levels, SPT results, and AD severity were available to assess the significance of later results, changes over time in these variables, or both on outcomes of milk allergy (see Table E2 in this article's Online Repository at www.jacionline.org). For both milk-specific IgE level and SPT wheal size, the most recent assessments were also highly significant $(P<.001)$ predictors of milk allergy resolution, whereas the associated baseline measure did not significantly contribute to the bivariate model. For these variables, the current status is therefore also important for prognosis. In contrast, the most recent $\mathrm{AD}$ score was not additionally predictive of milk allergy resolution when adjusted for the baseline value.

With regard to other atopic diseases, resolution was not associated with other food allergy at any time point. Resolution was slightly less likely in those who had asthma and rhinitis with hazard ratios for the time-varying most recent status covariate of $0.639(P=.043)$ for asthma and $0.646(P=.022)$ for rhinitis, suggesting that the development of these other atopic diseases might be associated with a decreased chance of resolution as children age. Not surprisingly, in a 2-variable time-varying covariate analysis, having baked milk without a reaction increased the chance of resolution (relative hazard, $4.1 ; P<.0001$ ), whereas having a reaction decreased the chance of resolution (relative hazard, $0.28 ; P=.072$ ).

T-cell studies assessed at baseline demonstrated no relationship between casein-stimulated expression of cytokine-inducible SH2-containing protein, forkhead box protein 3, GATA3, T-bet, IL-10, IL-4, and/or IFN- $\gamma$ and the resolution of milk allergy (data not shown). Analyses of serial measurements of these markers also did not identify any significant predictor of milk allergy resolution.

Finally, we used the 3 baseline factors most predictive of milk allergy resolution to develop a composite score that could be applied to individual patients (see Table E3 in this article's Online Repository at www.jacionline.org). For example, as represented in Fig 5, the likelihood of milk allergy resolution for 3 individual patients is predicted by using a composite index incorporating their milk-specific IgE levels, SPT wheal sizes, and severities of $\mathrm{AD}$, with lower limits for IgE set at $0.35 \mathrm{kU}_{\mathrm{A}} / \mathrm{L}$ and SPT response at $2.5 \mathrm{~mm}$. The $\mathrm{C}$ index for the 3-variable model is 0.724 (0.019 SE), with the single baseline variable component models ranging from 0.665 for the presence of moderate or severe $\mathrm{AD}$ to 0.720 for log milk-specific IgE. We have provided a Web-based calculator that can be used in counseling individual families as to the possible prognosis of their young ( $<15$ months) child's milk allergy (see www.cofargroup.org).

\section{DISCUSSION}

In this report we have described the natural history of milk allergy in a cohort of children enrolled in an observational study with a diagnosis of milk allergy. The study demonstrates a resolution rate of just more than $50 \%$ through age 5 years, which falls somewhere in the middle of previously published studies. ${ }^{3-16}$

Review of those prior studies reveals 2 salient points as to why interstudy results can differ so widely. First, results of studies from general populations are more likely to show a more favorable prognosis compared with studies of children from referral populations, as are prospective studies, especially those that include regular evaluations. For example, in a Danish population-based study, $76 \%$ of infants with milk allergy had become tolerant by age 
3 years $^{2}$ compared with only $19 \%$ by age 4 years and $42 \%$ by age 8 years in a retrospective study of a population referred to a tertiary care center. ${ }^{14}$ It is therefore consistent that our results fall between these extremes in that although the analysis was prospective, it did include a population preselected for milk or egg allergy who were evaluated at tertiary referral centers.

As has been demonstrated in several previous studies, ${ }^{3-10,12-14,16}$ both milk-specific IgE and SPT results were highly predictive of outcome. This was even the case at baseline, and these associations persisted through the period of observation. The relationship of AD to the natural course of milk allergy has been less clear from prior studies, but in this analysis moderate-to-severe $\mathrm{AD}$ at baseline was highly predictive of persistent disease. There was also a relationship of borderline significance to coexisting asthma and rhinitis by the end of the observation period. However, no relationships were noted to milk-specific $\mathrm{IgG}_{4}, \mathrm{IgE} /$ $\mathrm{IgG}_{4}$ ratio, or casein-stimulated T-cell studies.

The substantial predictive capacity of milk-specific IgE, SPT wheal size, and AD severity allowed for the development of a novel algorithm to estimate the natural course of milk allergy. This composite index has been developed into an equation that can be applied to young ( $<15$ months) patients presenting to the clinic and has been provided as a Web-based calculator, as well as a computer application. We believe that this unique tool will benefit health care providers and patients in providing early guidance as to the likelihood for disease resolution or persistence.

The strengths of this study include the sample size, the prospective design with re-evaluation at regular intervals, the inclusion of multiple research sites, and the exceptional follow-up rate. In addition, this study was the first to include detailed analysis of milk-specific $\mathrm{IgG}_{4}$ levels, as well as casein-stimulated T-cell cytokine responses, at baseline and follow-up. Although it was somewhat surprising that these studies provided no additional insight into the immunologic basis for the natural acquisition of milk tolerance, they are an important contribution to the literature.

Our results are somewhat limited by the fact that oral food challenges were not performed at protocol-defined intervals in this observational study and that many children were deemed milk tolerant based on unsupervised home introductions. In addition, the reliability of our algorithm might differ if different methods are used for SPT and IgE measurements. Finally, although we clearly demonstrated a relationship between the natural history of milk allergy and $\mathrm{AD}$ severity, we did this by using an $\mathrm{AD}$ assessment tool that is not routinely used in clinical practice. This tool was chosen, recognizing that there is no ideal method for AD assessment ${ }^{28}$ but that the Rajka-Langeland tool has the advantage of assessing diseases activity over time rather than as a single assessment based entirely on physical findings. As such, we do believe that the 3 -step gradation provided by this scale is very likely to correlate with the typical clinical assessment of AD severity.

An additional limitation was that we did not characterize baked milk consumption in a rigorous manner, although approximately $20 \%$ of those designated as having milk allergy in our cohort reported consumption of products with baked milk without a reaction. The ability of a subset of children with milk allergy to consume products with extensively heated milk appears to be associated with a phenotype of milk allergy that is more likely to resolve, and resolution can be accelerated by ingesting these foods. ${ }^{29,30}$ Therefore it is important to recognize that our overall estimate of resolution does not include at least some children who might be fully tolerant of even unheated milk or examine whether the introduction of baked milk might have influenced the natural course of milk allergy in this cohort. Nonetheless, 
the study did not specifically encourage trial or oral food challenge to baked milk products, and therefore we believe the results reflect clinical practice.

In conclusion, we estimate from this well-characterized cohort that approximately $50 \%$ of children with milk allergy will become milk tolerant by 5 years of age. Resolution is highly associated with lower milk-specific IgE levels, smaller SPT wheal sizes, and the absence of significant AD. These highly predictive variables have been used to provide the clinician with a calculator to predict the natural history of milk allergy for individual patients under their care, although additional studies to validate the model will be needed.

\section{Supplementary Material}

Refer to Web version on PubMed Central for supplementary material.

\section{Acknowledgments}

Additional site investigators: F. M. Atkins, D. Y. M. Leung, T. T. Perry, and A. M. Scurlock.

Coordinators and support: D. Brown, L. Talarico, S. Noone, K. Mudd, S. Knorr, P. Steele, J. Kamilaris, S. Carlisle, P. Mayfield, M. Mishoe, H. Haczynska, J. Grabowska, A. Hiegel, J. Straw, L. Christie, M. Groetch, J. Ellingson, J. Stone, S. Leung, K. Morgan, and K. Brown-Engelhardt.

We thank Dr Marshall Plaut, the medical officer, and J. Poyser for managing the project for the Consortium of Food Allergy Research (National Institute of Allergy and Infectious Diseases). We thank the families who kindly participated. We thank the staff of the clinical research units at each institution and the Statistical and Clinical Coordinating Center, without whose participation the study could not have been done. We thank Greer (Lenoir, NC) and Thermo Fisher Scientific (Waltham, MA) for supplying reagents.

Supported by National Institutes of Health (NIH)/National Institute of Allergy and Infectious Diseases grants U19AI066738 and U01AI066560. The project was also supported by grant nos. UL1 RR025780 (National Jewish), UL1 1TR000067 (Mount Sinai), UL 1 TR000039 (Arkansas), UL 1 RR024128 (North Carolina) and UL1 RR 025005 (Johns Hopkins) from the National Center for Research Resources (NCRR), a component of the NIH. Its contents are solely the responsibility of the authors and do not necessarily represent the official view of the NCRR or the NIH.

R. A. Wood has consultant arrangements with the Asthma and Allergy Foundation of America, is employed by Johns Hopkins University, has received research support from the National Institutes of Health (NIH), and receives royalties from UpToDate. S. H. Sicherer has received grants from the NIH/National Institute of Allergy and Infectious Diseases (NIAID) and has consultant arrangements with the Food Allergy Initiative. B. P. Vickery has received grants and travel support from the NIH/NIAID; has consultant arrangements with Mead Johnson; has received grants from the NIH/NIAID, the Thrasher Research Fund, the American Lung Association, and Cephalon. S. M. Jones is a member of the medical advisory board for the Food Allergy \& Anaphylaxis Network (FAAN); has received grants from the NIH, the FAAN, and the National Peanut Board; has received payment for lectures, including service on speakers' bureaus, from Abbott Nutrition International, the Kentucky Society for Allergy, Asthma \& Immunology, the New England Allergy Society, the American College of Allergy, Asthma \& Immunology, Indiana University Medical School and Riley Children's Hospital, the Spanish Society of Allergy \& Clinic Immunology, and the Oregon Allergy Asthma \& Immunology Society; has served on the NIAID Safety Monitoring Committee, the Arkansas Medicaid Drug Review Committee, and the NIAID Study Section. D. M. Fleischer has received grants from the NIH/NIAID, has consultant arrangements with Sanofi-Aventis, and receives royalties from UpToDate. A. K. Henning has received grants from the NIH. A. W. Burks has received grants or has grants pending from the NIH, the Department of Defense, and the Wallace Research Foundation; is a board member for the American Academy of Allergy, Asthma \& Immunology, the NIH Hypersensitivity, Autoimmune, and Immune-mediated Disease Study Section, the Journal of Allergy and Clinical Immunology, and the US Food and Drug Administration; is on advisory boards for ExploraMed Development, LLC, the FAAN, and ActoGeniX; has consultant arrangements with Merck \& Co, Novartis Pharma AG, the Dannon Company, McNeill Nutritionals, and Schering-Plough; is employed by UNC Children's Hospital and Duke University; has received payment for lectures from Mylan Specialty; has received royalties from UpToDate; has received payment for development of educational presentations from Current Views; has stock/stock options in Allertein, Mastcell Pharmaceuticals, and Dow AgroSciences; and has received travel expenses from the European Academy of Allergy \& Clinical Immunology. A. Grishin has received grants from the NIH/NIAID and has consultant arrangements with Allertein Therapeutics. D. Stable in has received grants from the NIH. H. A. Sampson has received grants from the NIH and the NIAID, has received travel support from the NIAID, is on the Danone Scientific Advisory Board, has consultant 
arrangements with Allertein Therapeutics and the Food Allergy Initiative, is employed by Mount Sinai Medical School, and has received royalties from Elsevier-Wiley and UpToDate.

\section{References}

1. Boyce JA, Assa'ad A, Burks AW, Jones SM, Sampson HA, Wood RA, et al. Guidelines for the Diagnosis and Management of Food Allergy in the United States: report of the NIAID-sponsored expert panel. J Allergy Clin Immunol. 2010; 126(suppl):S1-58. [PubMed: 21134576]

2. Sicherer SH. Epidemiology of food allergy. J Allergy Clin Immunol. 2011; 127:594-602. [PubMed: 21236480]

3. Bishop JM, Hill DJ, Hosking CS. Natural history of cow milk allergy: clinical outcome. J Pediatr. 1990; 116:862-7. [PubMed: 2348289]

4. Businco L, et al. Chronic diarrhea due to cow's milk allergy. A 4- to 10-year follow-up study. Ann Allergy. 1985; 55:844-7. [PubMed: 4073603]

5. Dannaeus A, Johansson SG. A follow-up study of infants with adverse reactions to cow's milk. I. Serum IgE, skin test reactions and RAST in relation to clinical course. Acta Paediatr Scand. 1979; 68:377-82. [PubMed: 571664]

6. Elizur A, et al. Natural course and risk factors for persistence of IgE-mediated cow's milk allergy. J Pediatr. 2012; 161:482-7.e1. [PubMed: 22480700]

7. Fiocchi A, et al. Incremental prognostic factors associated with cow's milk allergy outcomes in infant and child referrals: the Milan Cow's Milk Allergy Cohort study. Ann Allergy Asthma Immunol. 2008; 101:166-73. [PubMed: 18727472]

8. Hill DJ, et al. Recovery from milk allergy in early childhood: antibody studies. J Pediatr. 1989; 114:761-6. [PubMed: 2715889]

9. Hill DJ, et al. Natural history of cows' milk allergy in children: immunological outcome over 2 years. Clin Exp Allergy. 1993; 23:124-31. [PubMed: 8448679]

10. Hill DJ, et al. Manifestations of milk allergy in infancy: clinical and immunologic findings. $\mathbf{J}$ Pediatr. 1986; 109:270-6. [PubMed: 3734964]

11. Host A, Halken S. A prospective study of cow milk allergy in Danish infants during the first 3 years of life. Clinical course in relation to clinical and immunological type of hypersensitivity reaction. Allergy. 1990; 45:587-96. [PubMed: 2288394]

12. Host A, et al. The natural history of cow's milk protein allergy/intolerance. Eur J Clin Nutr. 1995; 49(suppl 1):S13-8. [PubMed: 8647059]

13. James JM, Sampson HA. Immunologic changes associated with the development of tolerance in children with cow milk allergy. J Pediatr. 1992; 121:371-7. [PubMed: 1517910]

14. Saarinen KM, et al. Clinical course and prognosis of cow's milk allergy are dependent on milkspecific IgE status. J Allergy Clin Immunol. 2005; 116:869-75. [PubMed: 16210063]

15. Skripak JM, et al. The natural history of IgE-mediated cow's milk allergy. J Allergy Clin Immunol. 2007; 120:1172-7. [PubMed: 17935766]

16. Vanto T, et al. Prediction of the development of tolerance to milk in children with cow's milk hypersensitivity. J Pediatr. 2004; 144:218-22. [PubMed: 14760265]

17. Sicherer SH, et al. Immunologic features of infants with milk or egg allergy enrolled in an observational study (Consortium of Food Allergy Research) of food allergy. J Allergy Clin Immunol. 2010; 125:1077-83.e8. [PubMed: 20451041]

18. Garcia-Ara C, et al. Specific IgE levels in the diagnosis of immediate hypersensitivity to cows' milk protein in the infant. J Allergy Clin Immunol. 2001; 107:185-90. [PubMed: 11150010]

19. Rajka G, Langeland T. Grading of the severity of atopic dermatitis. Acta Derm Venereol Suppl (Stockh). 1989; 144:13-4. [PubMed: 2800895]

20. Kull I, et al. Breast feeding and allergic diseases in infants-a prospective birth cohort study. Arch Dis Child. 2002; 87:478-81. [PubMed: 12456543]

21. Leung DY, et al. Disease management of atopic dermatitis: an updated practice parameter. Joint Task Force on Practice Parameters. Ann Allergy Asthma Immunol. 2004; 93(suppl 2):S1-21. [PubMed: 15478395] 
22. Food allergy: a practice parameter. Ann Allergy Asthma Immunol. 2006; 96(suppl 2):S1-68. [PubMed: 16597066]

23. American Academy of Pediatrics. Committee on Nutrition. Hypoallergenic infant formulas. Pediatrics. 2000; 106:346-9. [PubMed: 10920165]

24. Fleischer DM, et al. Allergic reactions to foods in preschool-aged children in a prospective observational food allergy study. Pediatrics. 2012; 130:e25-32. [PubMed: 22732173]

25. Clark TG, et al. Survival analysis part I: basic concepts and first analyses. Br J Cancer. 2003; 89:232-8. [PubMed: 12865907]

26. Harrell FE Jr, Lee KL, Mark DB. Multivariable prognostic models: issues in developing models, evaluating assumptions and adequacy, and measuring and reducing errors. Stat Med. 1996; 15:361-87. [PubMed: 8668867]

27. Pencina MJ, D'Agostino RB. Overall C as a measure of discrimination in survival analysis: model specific population value and confidence interval estimation. Stat Med. 2004; 23:2109-23. [PubMed: 15211606]

28. Schmitt J, Spuls P, Boers M, Thomas K, Chalmers J, Roekevisch E, et al. Towards global consensus on outcome measures for atopic eczema research: results of the HOME II meeting. Allergy. 2012; 67:1111-7. [PubMed: 22844983]

29. Kim JS, et al. Dietary baked milk accelerates the resolution of cow's milk allergy in children. J Allergy Clin Immunol. 2011; 128:125-31. e2. [PubMed: 21601913]

30. Nowak-Wegrzyn A, et al. Tolerance to extensively heated milk in children with cow's milk allergy. J Allergy Clin Immunol. 2008; 122:342-7. e1-2. [PubMed: 18620743]

\section{Abbreviations used}
AD Atopic dermatitis
Ct Cycle threshold
SPT Skin prick test 
Clinical implications: Milk allergy is outgrown in a majority of children by school age. Baseline milk IgE levels and SPT response sizes, along with AD severity, can be used to predict the natural course of disease. 


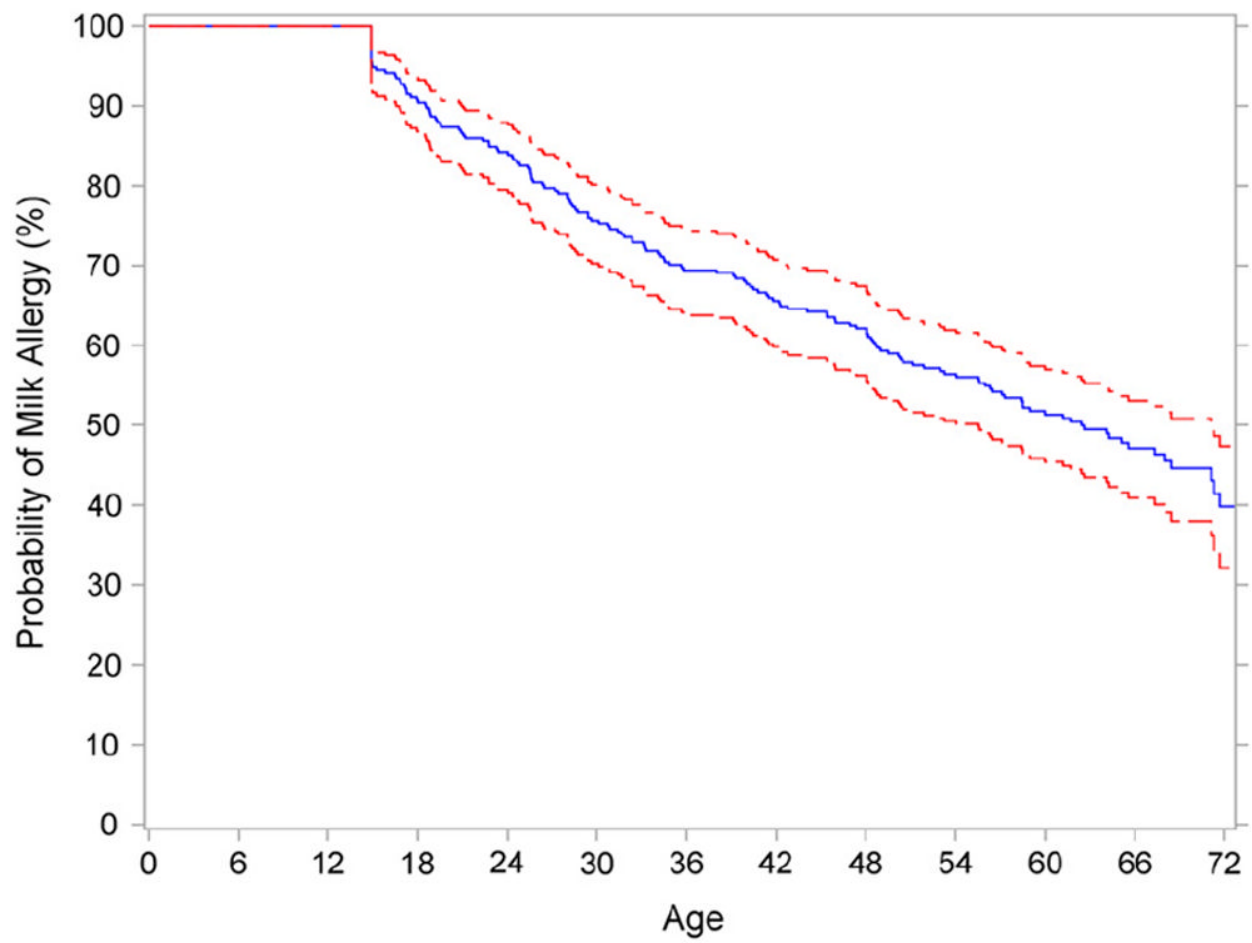

FIG 1.

Kaplan-Meier analysis of milk allergy resolution over time is shown in blue, with pointwise 95\% CIs shown in red. 


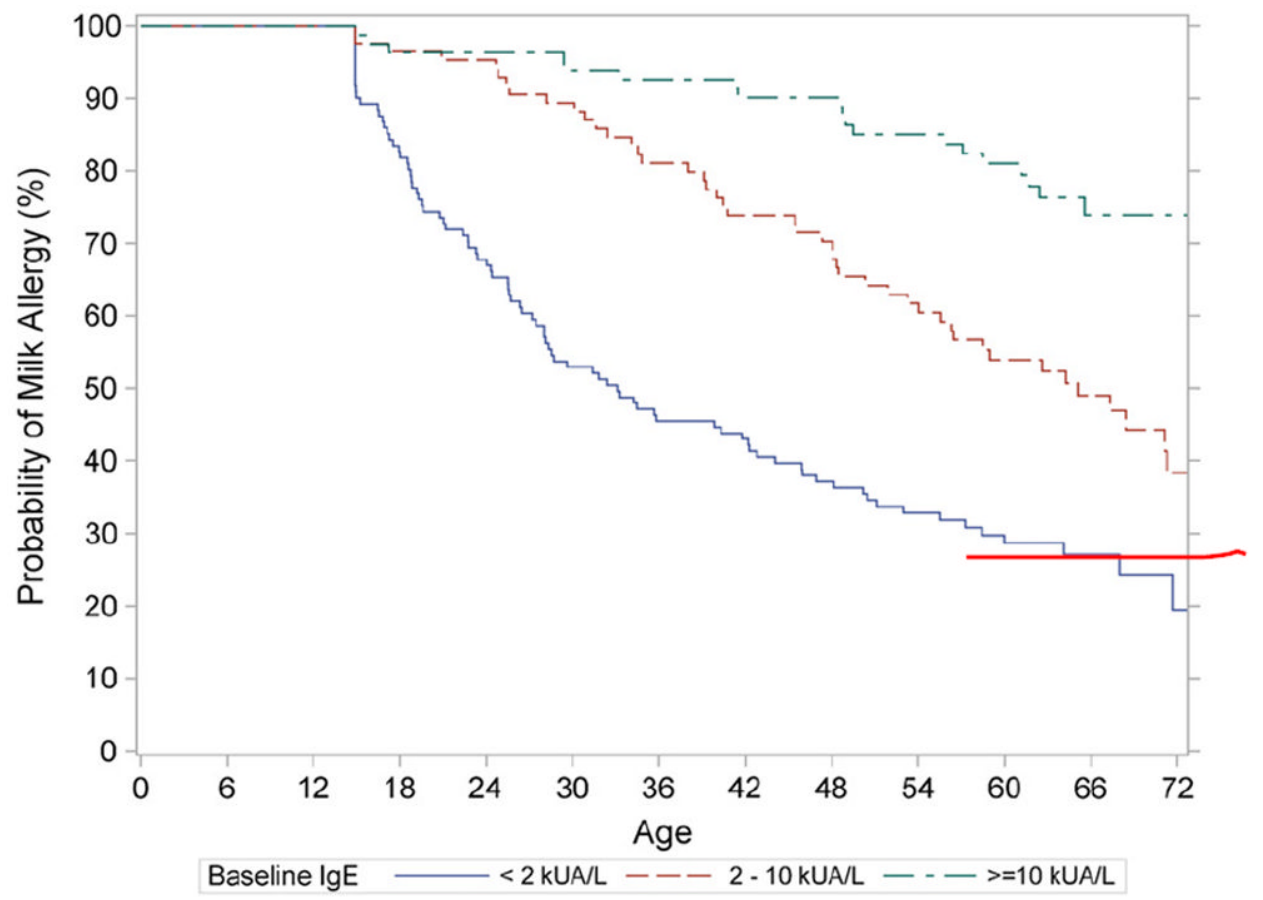

FIG 2.

Kaplan-Meier analysis representing the relationship of milk allergy resolution to baseline milk-specific IgE levels. Individual curves represent $\operatorname{IgE}$ levels of less than $2 \mathrm{kU}_{\mathrm{A}} / \mathrm{L}$ (blue), 2 to $10 \mathrm{kU}_{\mathrm{A}} / \mathrm{L}$ (red), and $10 \mathrm{kU}_{\mathrm{A}} / \mathrm{L}$ or greater (green). 


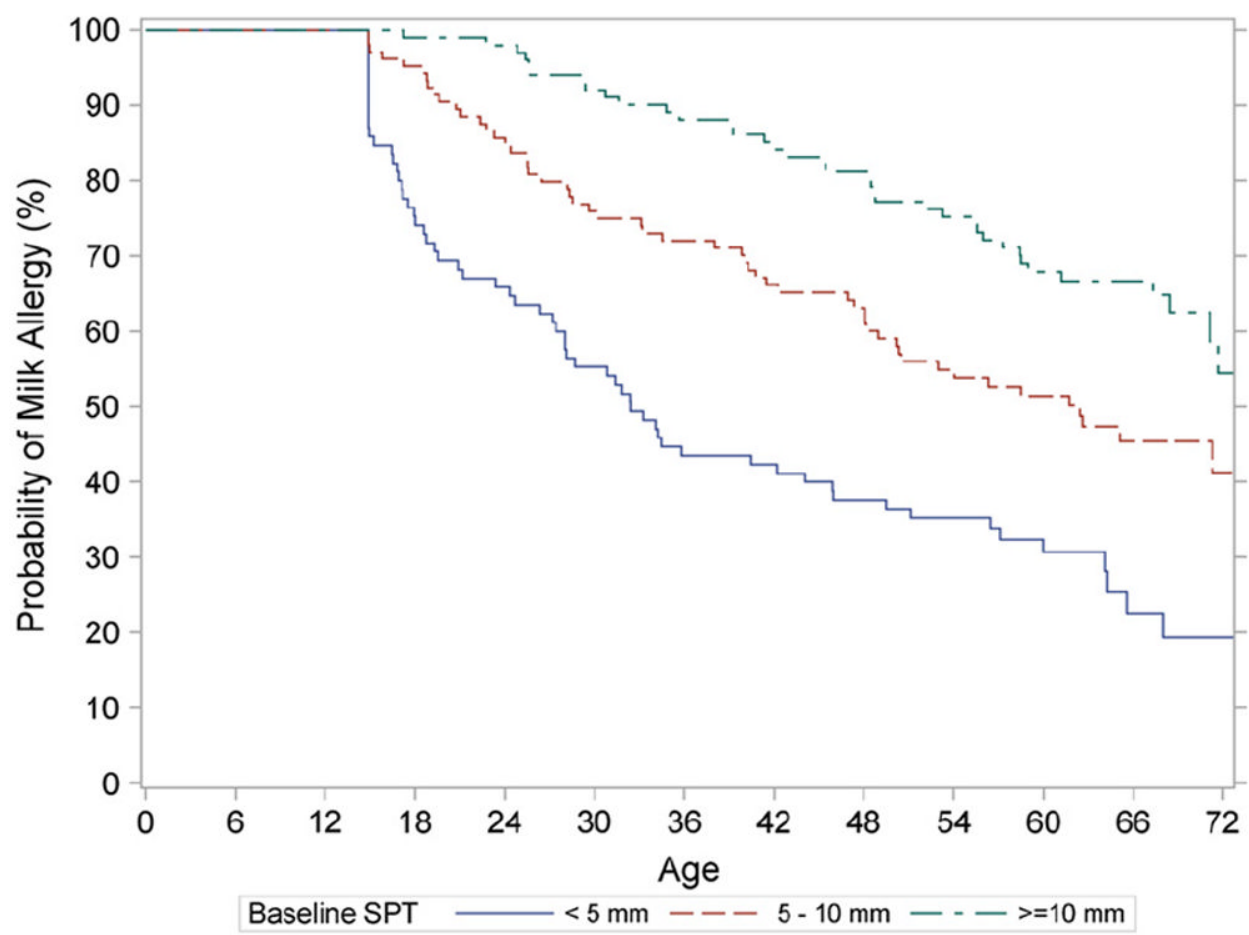

FIG 3.

Kaplan-Meier analysis representing the relationship of milk allergy resolution to baseline milk SPT wheal size. Individual curves represent wheal sizes of less than $5 \mathrm{~mm}$ (blue), 5 to $10 \mathrm{~mm}$ (red), and greater than $10 \mathrm{~mm}$ (green). 


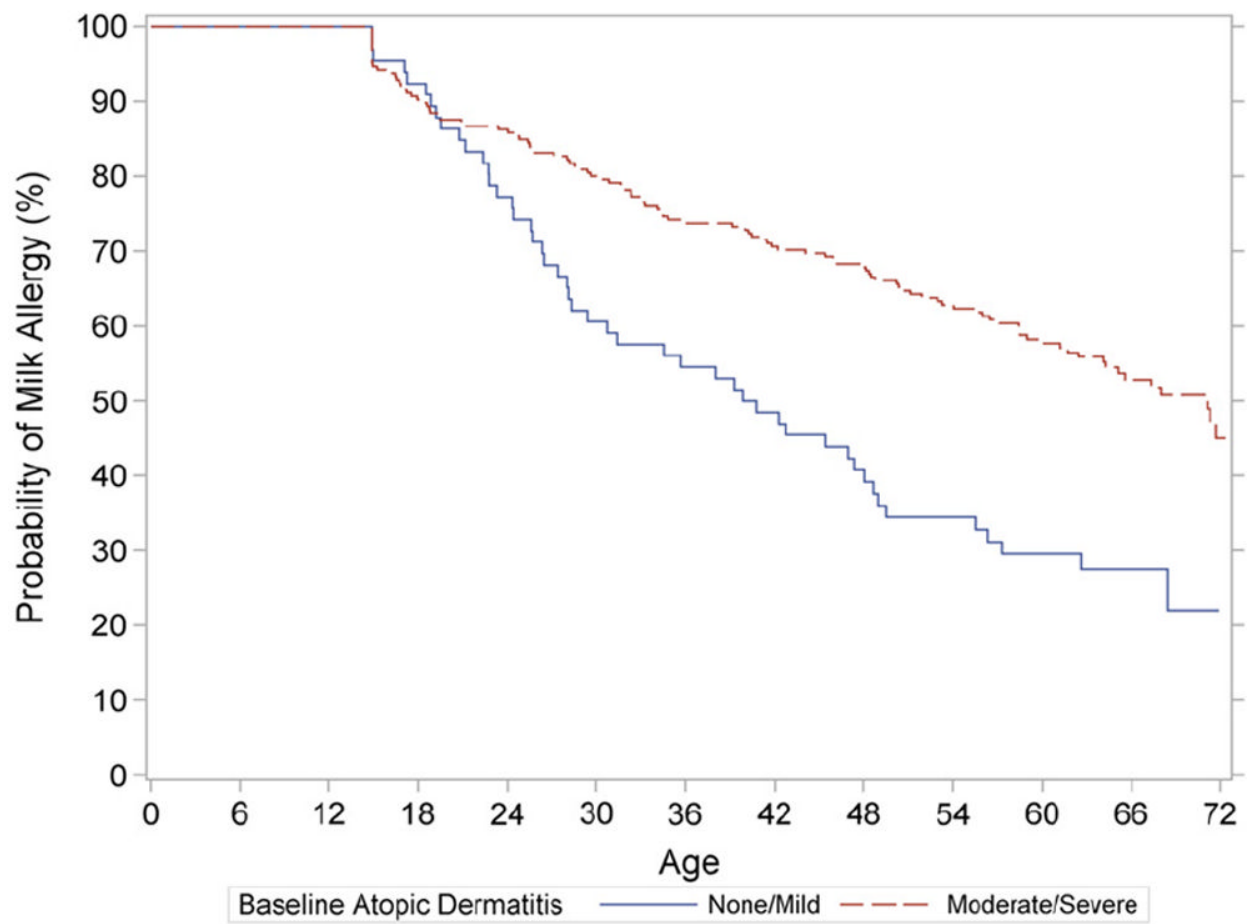

FIG 4.

Kaplan-Meier analysis representing the relationship of milk allergy resolution to baseline $\mathrm{AD}$. Individual curves represent no/mild AD (blue) and moderate/severe AD (red). 


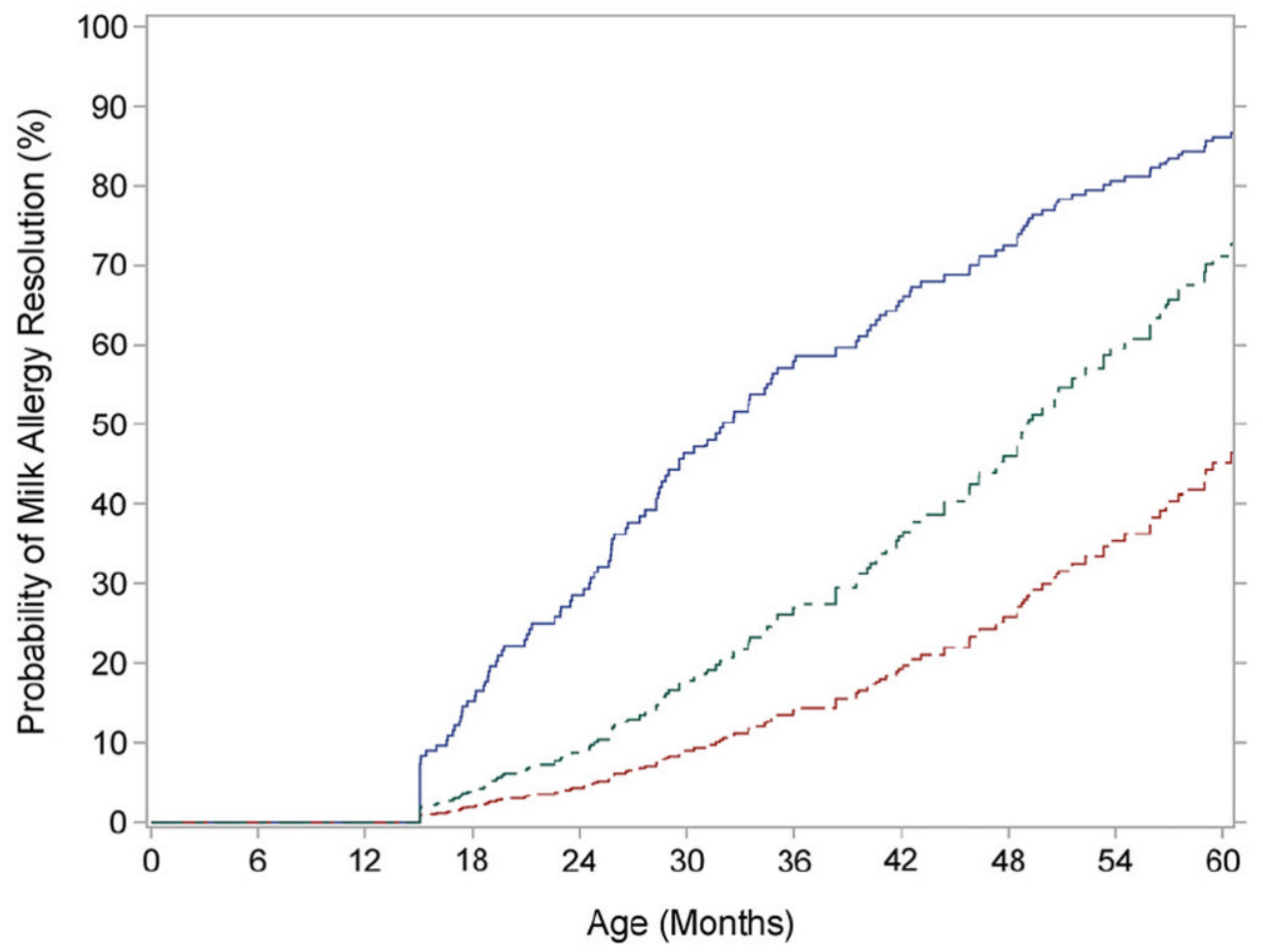

FIG 5.

This figure represents results of a composite index based on the baseline milk-specific IgE level, SPT wheal, and severity of AD, which can be used to estimate the likelihood of milk allergy resolution. For example, the lower curve (red) in this figure represents a patient with a milk-specific $\mathrm{IgE}$ level of $20 \mathrm{kU} / \mathrm{L}$, an SPT mean wheal diameter of $7 \mathrm{~mm}$, and moderate-to-severe $\mathrm{AD}$, whereas the middle curve (green) represents a patient with the same IgE level and skin test score but no or mild AD. The upper curve (blue) represents another patient with a milk IgE level of $2 \mathrm{kU}_{\mathrm{A}} / \mathrm{L}$, an SPT wheal score of $4 \mathrm{~mm}$, and no or mild AD. 


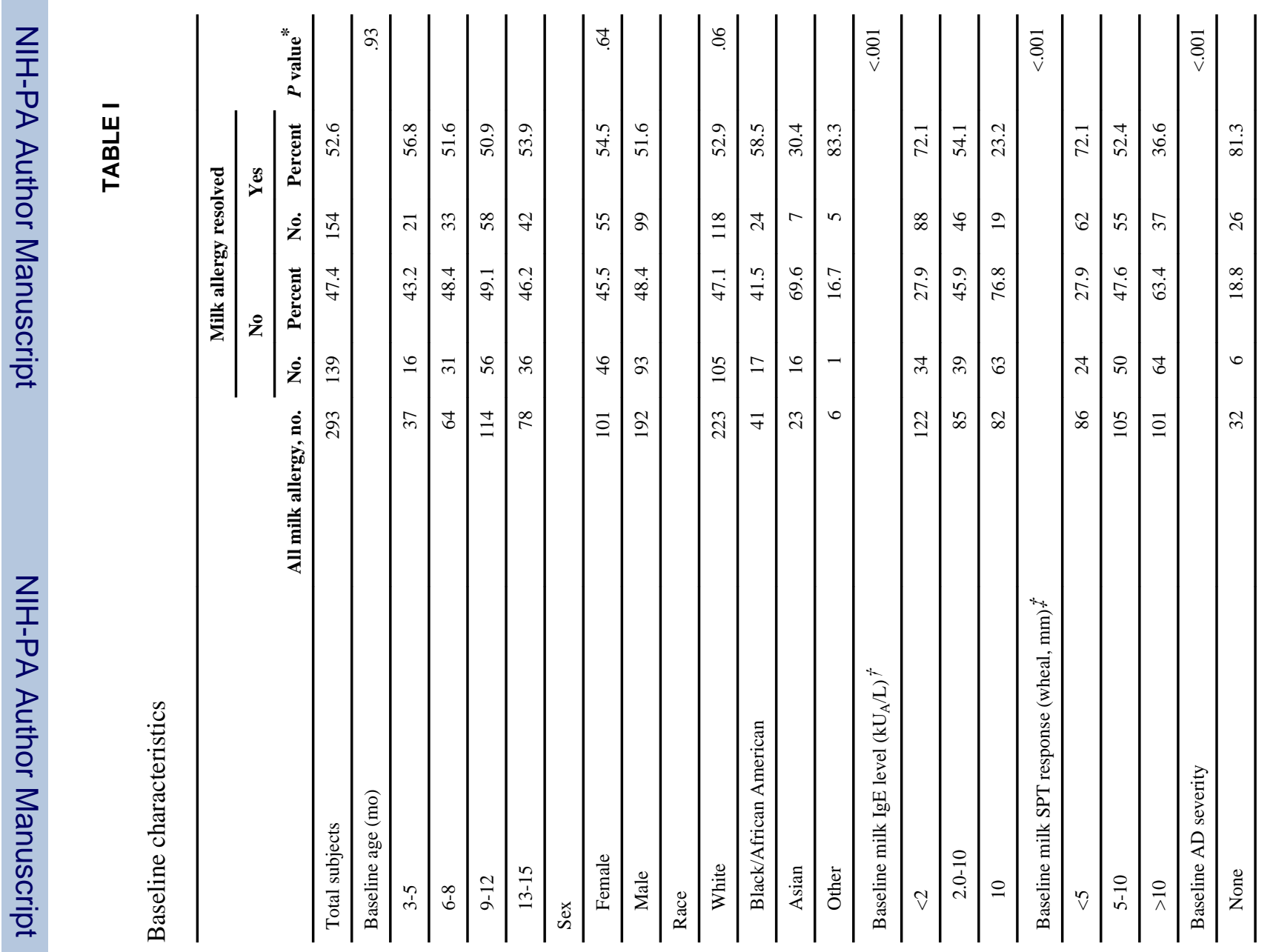




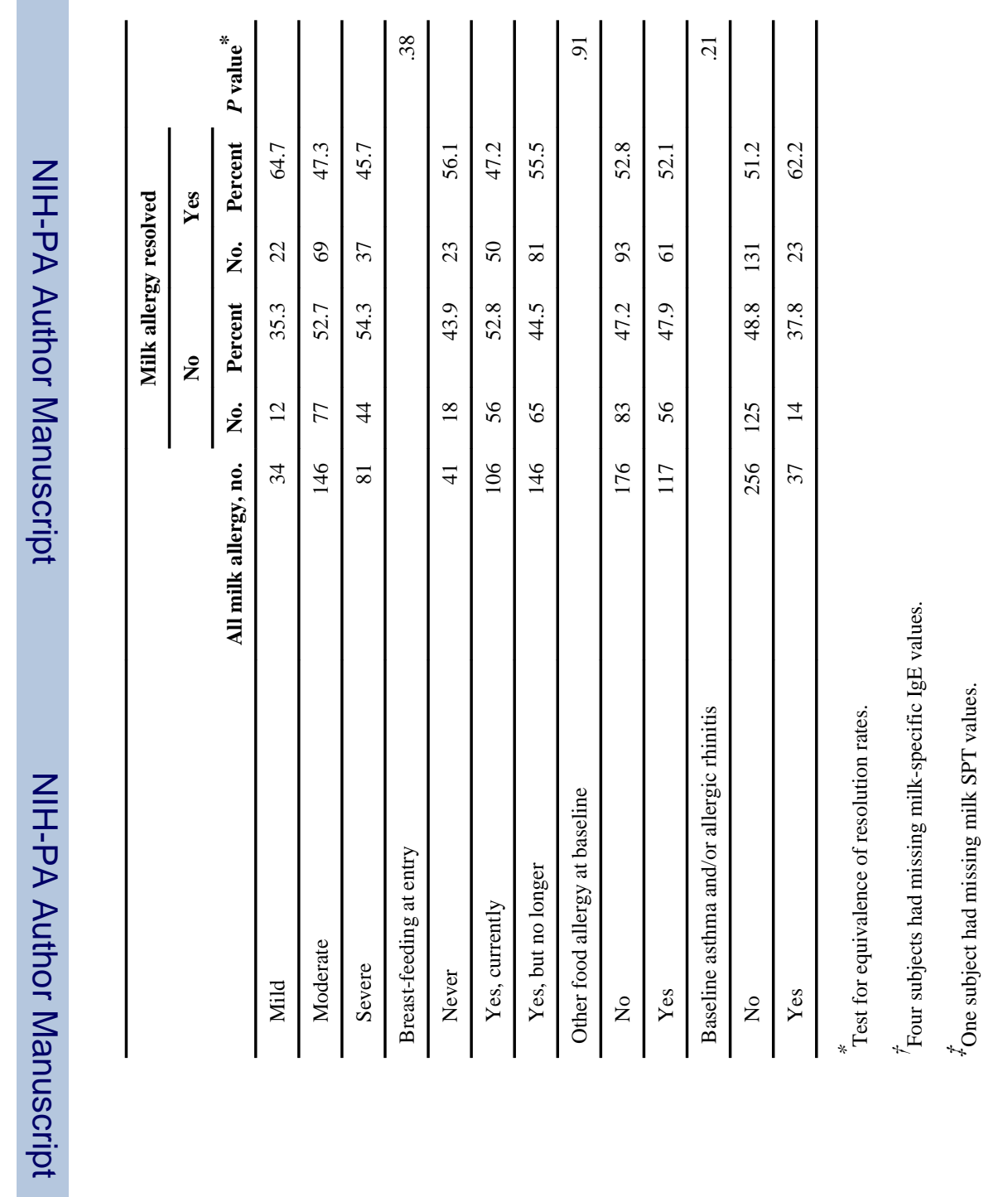

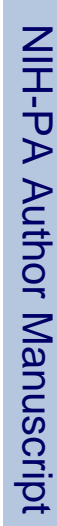


TABLE II

Resolution of milk allergy (Cox regression analysis with 1 variable in the model at a time)

\begin{tabular}{|c|c|c|c|}
\hline Factor for resolution of milk allergy & Hazard ratio & 95\% Hazard ratio confidence limits & $P$ value* \\
\hline \multicolumn{4}{|l|}{ Baseline age (mo) } \\
\hline $3-5$ vs $13-15$ & 1.40 & $0.82-2.36$ & .378 \\
\hline $6-8$ vs $13-15$ & 1.12 & $0.71-1.76$ & \\
\hline $9-12$ vs $13-15$ & 0.91 & $0.61-1.35$ & \\
\hline \multicolumn{4}{|l|}{ Sex } \\
\hline Female vs male & 1.28 & $0.92-1.78$ & .141 \\
\hline \multicolumn{4}{|l|}{ Race } \\
\hline White vs nonwhite & 1.02 & $0.70-1.48$ & .909 \\
\hline \multicolumn{4}{|l|}{ Baseline milk-specific IgE level $\left(\mathrm{kU}_{\mathrm{A}} / \mathrm{L}\right)$} \\
\hline$<2$ vs $\geq 10$ & 5.74 & $3.48-9.46$ & $<.001$ \\
\hline $2-<10$ vs $\geq 10$ & 2.66 & $1.56-4.54$ & \\
\hline \multicolumn{4}{|l|}{ Baseline AD } \\
\hline Mild/none vs moderate/severe & 2.09 & $1.48-2.94$ & $<.001$ \\
\hline \multicolumn{4}{|l|}{ Baseline milk SPT response (mm) } \\
\hline$<5$ vs $>10$ & 3.65 & $2.42-5.51$ & $<.001$ \\
\hline $5-10$ vs $>10$ & 1.86 & $1.22-2.82$ & \\
\hline \multicolumn{4}{|l|}{ Breast-feeding at entry } \\
\hline Yes, but no longer vs never & 0.94 & $0.59-1.49$ & .399 \\
\hline Yes, currently vs never & 0.76 & $0.46-1.24$ & \\
\hline \multicolumn{4}{|l|}{ Other food allergy } \\
\hline Yes vs none & 0.94 & $0.68-1.29$ & .687 \\
\hline \multicolumn{4}{|l|}{ Asthma or rhinitis } \\
\hline Yes vs none & 1.30 & $0.83-2.03$ & .246 \\
\hline
\end{tabular}

